Copyright by the Acoustical Society of America. Yang, J. S., \& Batra, R. C. (1995). A second-order theory for piezoelectric materials. Journal of the Acoustical Society of America, 97(1), 280-288. doi: 10.1121/1.412312

\title{
A second-order theory for piezoelectric materials
}

\author{
J. S. Yang \\ Department of Mechanical Engineering, Rensselaer Polytechnic Institute, Troy, New York 12180 \\ R. C. Batra \\ Department of Engineering Science and Mechanics, Virginia Polytechnic Institute and State University, \\ Blacksburg, Virginia 24061-0219
}

(Received 22 February 1994; revised 1 July 1994; accepted 8 August 1994)

\begin{abstract}
Based on the theory of invariants, from invariant polynomial constitutive relations for piezoelectric materials which are either transversely isotropic or are of class $m m 2$ are derived from the polynomial integrity basis functions. These constitutive relations are assumed to be smooth enough functions of their arguments to be expanded in terms of a Taylor series. These functions are expanded about the values their arguments take in the reference configuration and all terms up to the quadratic terms in the gradients of the mechanical displacement and electric potential are kept. The second-order theory so obtained is then specialized to the case of small deformations and weak electric fields, and the case of small deformations and relatively strong electric fields. The material parameters in the present theory are identified by relating them to those in the more conventional theories.
\end{abstract}

PACS numbers: $43.40 . A t$, 43.25.Ba

\section{INTRODUCTION}

The effect of nonlinearity in the constitutive relations of piezoelectric ceramics has been of recent interest. Lazarus and Crawley ${ }^{1}$ have pointed out that piezoelectric strain "constants" are not constants but depend on the induced strains. Norwood et al., ${ }^{2}$ Kulkarni and Hanagud ${ }^{3,4}$ used a NeoHookean constitutive relation to model the response of piezoelectric ceramics. The Neo-Hookean model is an affine relationship between the second Piola-Kirchhoff stress tensor and the Green-Lagrange strain tensor. It contains some, but not all of the quadratic terms of the mechanical displacement gradient and the electric potential gradient with respect to the reference material coordinates. Pai et al. ${ }^{5}$ also considered the dependence of the piezoelectric strain parameters upon the strain in formulating a plate theory of piezoelectric laminates. Tiersten ${ }^{6}$ derived a general form for electroelastic equations cubic in the mechanical displacement gradient and electric potential gradient and specialized it to isotropic elastic solids by the theory of invariants. ${ }^{7}$ Recently, Tiersten specialized his equations to the case of small deformations and large electric fields ${ }^{8}$ and developed the corresponding plate theory. ${ }^{9}$

Piezoelectric materials exhibiting transverse isotropy ${ }^{3,4}$ or of class $m m 2$ symmetry ${ }^{10,11}$ are widely used in transducers and smart structures. Varadan et al. ${ }^{12}$ have derived linear, form invariant constitutive relations for transversely isotropic piezoelectric materials by the theory of invariants. The derivation, based on the theory of invariants, automatically gives the minimum number of material parameters consistent with the material symmetry. These constitutive relations are useful in some problems involving fields and waves in anisotropic media. ${ }^{12}$

In this paper, based on the theory of invariants, form invariant polynomial constitutive relations for piezoelectric materials which are either transversely isotropic or are of class $m m 2$ are derived from the polynomial integrity basis functions. These constitutive relations are assumed to be smooth enough functions of their arguments to be expanded in terms of a Taylor series. These functions are expanded about the values their arguments take in the reference configuration and all terms up to the quadratic terms in the gradients of the mechanical displacement and the electric potential are kept. The resulting equations constitute the so-called second-order theory. ${ }^{13}$ The theory is then specialized to the case of small deformations and weak electric fields, and the case of small deformations and relatively strong electric fields. The material parameters in the present theory are then determined by relating them to those in the more conventional theories. The method can be used to obtain nonlinear constitutive relations for piezoelectric ceramics of other symmetries or containing higher-order terms of the mechanical displacement gradient and electric potential gradient.

\section{EQUATIONS FOR AN ELASTIC DIELECTRIC}

Let the coordinates of a material particle with respect to a rectangular Cartesian coordinate system be $X_{K}$ in the reference configuration, its spatial coordinates in the current configuration be $x_{k}$, then the equations for an elastic dielectric are ${ }^{14}$

$$
\begin{aligned}
& \left(T_{k l}+\epsilon_{0} E_{k} E_{l}-\frac{1}{2} \epsilon_{0} E_{m} E_{m} \delta_{k l}\right)_{, k}=\rho \dot{v}_{l}, \\
& \left(P_{k}+\epsilon_{0} E_{k}\right)_{, k}=0, \\
& T_{k l}=\rho \frac{\partial \Psi}{\partial E_{K L}} x_{k, K} x_{l, L}, \\
& P_{k}=-\rho \frac{\partial \Psi}{\partial W_{K}} x_{k, K},
\end{aligned}
$$

where $T_{k l}=\tau_{k l}+P_{k} E_{l}$ is a symmetric stress tensor called the elastic stress, $\tau_{k l}$ the Cauchy stress, $\rho$ the mass density, $v_{l}$ 
velocity vector, $P_{k}$ electric polarization, $E_{k}=-\phi_{, k}$ electric field, $\phi$ electric potential, $\epsilon_{0}$ permittivity of the free space, $\Psi\left(E_{K L}, W_{K}\right)$ an energy density function, and a dot above a quantity signifies its material time derivative. $E_{K L}$ is the Green-Lagrange strain tensor and $W_{K}$ the electric field in material form

$$
\begin{gathered}
E_{K L}=\frac{1}{2}\left(U_{K, L}+U_{L, K}+U_{M, K} U_{M, L}\right), \\
W_{K}=x_{k, K} E_{k}=-x_{k, K} \phi_{, k}=-\phi_{, K},
\end{gathered}
$$

where $U_{K}$ is the mechanical displacement vector. Throughout this paper, a repeated index implies summation over the range of the index, and a comma followed by $K(i)$ implies partial differentiation with respect to $X_{K}\left(x_{i}\right)$. With the introduction of the elastic second Piola-Kirchhoff stress tensor $T_{K L}$, the material electric polarization $\Pi_{K}$, and $\Sigma$ as

$$
T_{K L}=J X_{K, k} X_{L, l} T_{k l}, \quad \Pi_{K}=J X_{K, k} P_{k}, \quad \Sigma=\rho_{0} \Psi,
$$

where $\rho_{0}=\rho J$ is the mass density in the reference configuration, $J=\operatorname{det}\left(x_{k, K}\right)$, the constitutive relations $(1)_{3}$ and $(1)_{4}$ can be written as

$$
T_{K L}=\frac{\partial \Sigma}{\partial E_{K L}}, \quad \Pi_{K}=-\frac{\partial \Sigma}{\partial W_{K}} .
$$

The following relations are used to obtain $T_{K l}, T_{k l}$, and $P_{k}$ from $T_{K L}$ and $\Pi_{K}$

$$
\begin{aligned}
& T_{K l}=x_{l, L} T_{K L}, \quad T_{k l}=J^{-1} x_{k, K} T_{K l}=J^{-1} x_{k, K} x_{l, L} T_{K L}, \\
& P_{k}=J^{-1} x_{k, K} \Pi_{K},
\end{aligned}
$$

where $T_{K l}$ is the first elastic Piola-Kirchhoff stress tensor.

\section{TRANSVERSELY ISOTROPIC MATERIALS}

Piezoelectric ceramics like PZT-5 are transversely isotropic. $^{12}$

\section{A. Form invariant polynomial constitutive relations}

A transversely isotropic material is invariant under rotations about an axis and reflections about planes containing that axis. Let a be a unit vector along the axis of the rotational invariance, then any scalar polynomial function of the symmetric tensor $\mathbf{E}$ and vector $\mathbf{W}$ invariant under the above transformations must be a polynomial of the following invariants called the polynomial integrity basis ${ }^{15}$

$$
\begin{aligned}
& I_{1}=\mathbf{a} \cdot \mathbf{E} \cdot \mathbf{a}, \quad I_{2}=\operatorname{tr} \mathbf{E}, \quad I_{3}=\mathbf{a} \cdot \mathbf{W}, \\
& I I_{1}=\mathbf{a} \cdot \mathbf{E}^{2} \cdot \mathbf{a}, \quad I I_{2}=\operatorname{tr} \mathbf{E}^{2}, \\
& I I_{3}=\mathbf{W} \cdot \mathbf{W}, \quad I I_{4}=\mathbf{a} \cdot \mathbf{E} \cdot \mathbf{W}+\mathbf{W} \cdot \mathbf{E} \cdot \mathbf{a}, \\
& I I I_{1}=\operatorname{tr} \mathbf{E}^{3}, \quad I I I_{2}=\mathbf{W} \cdot \mathbf{E} \cdot \mathbf{W}, \\
& I I_{3}=\mathbf{a} \cdot \mathbf{E}^{2} \cdot \mathbf{W}+\mathbf{W} \cdot \mathbf{E}^{2} \cdot \mathbf{a},
\end{aligned}
$$

where $\mathbf{a} \cdot \mathbf{b}$ indicates the inner product between vectors $\mathbf{a}$ and $\mathbf{b}$, and $\operatorname{tr} \mathbf{E}$ equals the sum of the diagonal terms of $\mathbf{E}$. With (7), any scalar polynomial function of the symmetric tensor $\mathbf{E}$ and the vector $\mathbf{W}$ can be written as

$$
\Sigma=\Sigma\left(I_{1}, I_{2}, I_{3}, I I_{1}, I_{2}, I I_{3}, I_{4}, I I I_{1}, I I I_{2}, I I I_{3}\right),
$$

where $\Sigma$ is a general polynomial function of its arguments. From (5) and (8), we obtain the following general form for the polynomial constitutive relations for a transversely isotropic material

$$
\begin{aligned}
\mathbf{T}= & \frac{\partial \Sigma}{\partial I_{1}} \mathbf{a} \otimes \mathbf{a}+\frac{\partial \Sigma}{\partial I_{2}} \mathbf{1}+\frac{\partial \Sigma}{\partial I_{1}}(\mathbf{a} \otimes \mathbf{E} \cdot \mathbf{a}+\mathbf{a} \cdot \mathbf{E} \otimes \mathbf{a}) \\
& +2 \frac{\partial \Sigma}{\partial I I_{2}} \mathbf{E}+\frac{\partial \Sigma}{\partial I_{4}}(\mathbf{a} \otimes \mathbf{W}+\mathbf{W} \otimes \mathbf{a})+3 \frac{\partial \Sigma}{\partial I I_{1}} \mathbf{E}^{2} \\
& +\frac{\partial \Sigma}{\partial I I I_{2}} \mathbf{W} \otimes \mathbf{W}+\frac{\partial \Sigma}{\partial I I I_{3}}(\mathbf{a} \otimes \mathbf{E} \cdot \mathbf{W}+\mathbf{W} \cdot \mathbf{E} \otimes \mathbf{a} \\
& +\mathbf{W} \otimes \mathbf{E} \cdot \mathbf{a}+\mathbf{a} \cdot \mathbf{E} \otimes \mathbf{W}), \\
-\mathbf{\Pi}= & \frac{\partial \Sigma}{\partial I_{3}} \mathbf{a}+2 \frac{\partial \Sigma}{\partial I I_{3}} \mathbf{W}+2 \frac{\partial \Sigma}{\partial I_{4}} \mathbf{E} \cdot \mathbf{a}+2 \frac{\partial \Sigma}{\partial I I I_{2}} \mathbf{E} \cdot \mathbf{W} \\
& +2 \frac{\partial \Sigma}{\partial I I I_{3}} \mathbf{E}^{2} \cdot \mathbf{a},
\end{aligned}
$$

where $\mathbf{1}$ is the identity tensor and $\mathbf{u} \otimes \mathbf{v}$ denotes tensor product between the tensors $\mathbf{u}$ and $\mathbf{v}$. In order to derive a secondorder theory, we only keep all terms up to degree three in the polynomial function $\Sigma$

$$
\begin{aligned}
\Sigma= & \alpha_{1} I_{1}+\alpha_{2} I_{2}+\beta I_{3}+c_{1} I_{1}^{2}+c_{2} I_{2}^{2}+c_{3} I_{1} I_{2}+c_{4} I I_{1}+c_{5} I I_{2}+\epsilon_{1} I_{3}^{2}+\epsilon_{2} I I_{3}+e_{1} I_{1} I_{3}+e_{2} I_{2} I_{3}+e_{3} I I_{4}+\lambda_{1} I_{1}^{3}+\lambda_{2} I_{2}^{3}+\lambda_{3} I_{1}^{2} I_{2} \\
& +\lambda_{4} I_{2}^{2} I_{1}+\lambda_{5} I I_{1} I_{1}+\lambda_{6} I I_{1} I_{2}+\lambda_{7} I I_{2} I_{1}+\lambda_{8} I_{2} I_{2}+\lambda_{9} I I_{1}+\mu_{1} I_{3}^{2}+\mu_{2} I I_{3} I_{3}+\nu_{1} I_{1}^{2} I_{3}+\nu_{2} I_{3}^{2} I_{1}+\nu_{3} I_{2}^{2} I_{3}+\nu_{4} I_{3}^{2} I_{2} \\
& +\nu_{5} I I_{1} I_{3}+\nu_{6} I I_{2} I_{3}+\nu_{7} I I_{3} I_{1}+\nu_{8} I_{3} I_{2}+\nu_{9} I_{4} I_{1}+\nu_{10} I I_{4} I_{2}+\nu_{11} I_{4} I_{3}+\nu_{12} I I_{2}+\nu_{13} I I_{3}+\nu_{14} I_{1} I_{2} I_{3},
\end{aligned}
$$

where $\alpha_{1}$ and $\alpha_{2}$ will be shown to represent the initial stress, and $\beta$ the initial polarization. $c_{1}, c_{2}, c_{3}, c_{4}$, and $c_{5}$ are linear elastic constants, $\epsilon_{1}$ and $\epsilon_{2}$ linear dielectric constants, and $e_{1}, e_{2}$, and $e_{3}$ linear piezoelectric constants. $\lambda_{1}-\lambda_{9}, \mu_{1}, \mu_{2}$, and $\nu_{1}-\nu_{14}$ are constants for nonlinear materials. We note that there are ten constants for a linear material and 25 constants for a nonlinear material. With (10), Eq. (9) becomes 


$$
\begin{aligned}
\mathbf{T}= & \left(\alpha_{1}+2 c_{1} I_{1}+c_{3} I_{2}+e_{1} I_{3}+3 \lambda_{1} I_{1}^{2}+2 \lambda_{3} I_{1} I_{2}+\lambda_{4} I_{2}^{2}+\lambda_{5} I_{1}+\lambda_{7} I I_{2}+2 \nu_{1} I_{1} I_{3}+\nu_{2} I_{3}^{2}+\nu_{7} I_{3}+\nu_{9} I_{4}+\nu_{14} I_{2} I_{3}\right) \mathbf{a} \otimes \mathbf{a} \\
& +\left(\alpha_{2}+2 c_{2} I_{2}+c_{3} I_{1}+e_{2} I_{3}+3 \lambda_{2} I_{2}^{2}+\lambda_{3} I_{1}^{2}+2 \lambda_{4} I_{1} I_{2}+\lambda_{6} I_{1}+\lambda_{8} I_{2}+2 \nu_{3} I_{2} I_{3}+\nu_{4} I_{3}^{2}+\nu_{8} I_{3}+\nu_{10} I_{4}+\nu_{14} I_{1} I_{3}\right) \mathbf{1} \\
& +\left(c_{4}+\lambda_{5} I_{1}+\lambda_{6} I_{2}+\nu_{5} I_{3}\right)(\mathbf{a} \otimes \mathbf{E} \cdot \mathbf{a}+\mathbf{a} \cdot \mathbf{E} \otimes \mathbf{a})+2\left(c_{5}+\lambda_{7} I_{1}+\lambda_{8} I_{2}+\nu_{6} I_{3}\right) \mathbf{E}+\left(e_{3}+\nu_{9} I_{1}+\nu_{10} I_{2}+\nu_{11} I_{3}\right) \\
& \times(\mathbf{a} \otimes \mathbf{W}+\mathbf{W} \otimes \mathbf{a})+3 \lambda_{9} \mathbf{E}^{2}+\nu_{12} \mathbf{W} \otimes \mathbf{W}+\nu_{13}(\mathbf{a} \otimes \mathbf{E} \cdot \mathbf{W}+\mathbf{W} \cdot \mathbf{E} \otimes \mathbf{a}+\mathbf{W} \otimes \mathbf{E} \cdot \mathbf{a}+\mathbf{a} \cdot \mathbf{E} \otimes \mathbf{W}) \\
- & \mathbf{I}=\left(\beta+2 \epsilon_{1} I_{3}+e_{1} I_{1}+e_{2} I_{2}+3 \mu_{1} I_{3}^{2}+\mu_{2} I I_{3}+\nu_{1} I_{1}^{2}+2 \nu_{2} I_{3} I_{1}+\nu_{3} I_{2}^{2}+2 \nu_{4} I_{3} I_{2}+\nu_{5} I I_{1}+\nu_{6} I I_{2}+\nu_{11} I I_{4}+\nu_{14} I_{1} I_{2}\right) \mathbf{a} \\
& +2\left(\epsilon_{2}+\mu_{2} I_{3}+\nu_{7} I_{1}+\nu_{8} I_{2}\right) \mathbf{W}+2\left(e_{3}+\nu_{9} I_{1}+\nu_{10} I_{2}+\nu_{11} I_{3}\right) \mathbf{E} \cdot \mathbf{a}+2 \nu_{12} \mathbf{E} \cdot \mathbf{W}+2 \nu_{13} \mathbf{E}^{2} \cdot \mathbf{a} .
\end{aligned}
$$

Equations (11) and (12) are polynomial representations of $T$ and $\Pi$ of degree two in components of $\mathbf{E}$ and $\mathbf{W}$. It generalizes the Neo-Hookean-type constitutive relation ${ }^{2-4}$ and Varadan et al.'s results. ${ }^{12}$ It can be seen that terms in (11) involving $\alpha_{1}$ and $\alpha_{2}$ do not depend upon $\mathbf{E}$ or $\mathbf{W}$ and hence represent the initial stress. Similarly, the term involving $\beta$ in (12) represents the initial polarization which can only exist along the axis a of symmetry.

\section{B. Second-order theory}

By a second-order theory we mean a theory that contains all quadratic terms of the mechanical displacement gradient and electric potential gradient. Equations (11) and (12) contain some higher-order terms in this sense. To get a secondorder theory, we make the following decompositions

$$
\begin{aligned}
& \mathbf{E}=\mathbf{E}^{(1)}+\mathbf{E}^{(2)}, \quad E_{K L}^{(1)}=\frac{1}{2}\left(U_{K, L}+U_{L, K}\right), \\
& E_{K L}^{(2)}=\frac{1}{2} U_{M, K} U_{M, L}, \quad \mathbf{W}=\mathbf{W}^{(1)}, \quad W_{K}^{(1)}=-\phi_{, K}
\end{aligned}
$$

and expansions

$$
I_{1}=I_{1}^{(1)}+I_{1}^{(2)}, \quad I_{1}^{(1)}=\mathbf{a} \cdot \mathbf{E}^{(1)} \cdot \mathbf{a}, \quad I_{1}^{(2)}=\mathbf{a} \cdot \mathbf{E}^{(2)} \cdot \mathbf{a},
$$

$$
\begin{aligned}
& I_{2}=I_{2}^{(1)}+I_{2}^{(2)}, \quad I_{2}^{(1)}=\operatorname{tr} \mathbf{E}^{(1)}, \quad I_{2}^{(2)}=\operatorname{tr} \mathbf{E}^{(2)}, \\
& I_{3}=I_{3}^{(1)}, \quad I_{3}^{(1)}=\mathbf{W}^{(1)} \cdot \mathbf{a}, \\
& I I_{1}=I I_{1}^{(2)}+\ldots, \quad I I_{1}^{(2)}=\mathbf{a} \cdot\left(\mathbf{E}^{(1)}\right)^{2} \cdot \mathbf{a}, \\
& I I_{2}=I I_{2}^{(2)}+\ldots, \quad I I_{2}^{(2)}=\operatorname{tr}\left(\mathbf{E}^{(1)}\right)^{2}, \\
& I I_{3}=I I_{3}^{(2)}+\ldots, \quad I I_{3}^{(2)}=\mathbf{W}^{(1)} \cdot \mathbf{W}^{(1)}, \\
& I I_{4}=I I_{4}^{(2)}+\ldots, \quad I I_{4}^{(2)}=\mathbf{a} \cdot \mathbf{E}^{(1)} \cdot \mathbf{W}^{(1)}+\mathbf{W}^{(1)} \cdot \mathbf{E}^{(1)} \cdot \mathbf{a},
\end{aligned}
$$

where a superscript enclosed in parentheses indicates the order of the mechanical displacement and the electric potential in the quantity. We have written $\mathbf{W}$ as $\mathbf{W}^{(1)}$ to make formally superscripts of different terms homogeneous. Substituting (13) and (14) into (11) and (12), keeping terms up to second order, we obtain the following second-order representations for $\mathbf{T}$ and $\boldsymbol{I}$

$$
\begin{aligned}
& \mathbf{T}=\alpha_{1} \mathbf{a} \otimes \mathbf{a}+\alpha_{2} \mathbf{1}+\mathbf{T}^{(1)}+\mathbf{T}^{(2)}, \\
& \mathbf{\Pi}=-\beta \mathbf{a}+\Pi^{(1)}+\mathbf{\Pi}^{(2)},
\end{aligned}
$$

where

$$
\begin{aligned}
\mathbf{T}^{(1)}= & \left(2 c_{1} I_{1}^{(1)}+c_{3} I_{2}^{(1)}+e_{1} I_{3}^{(1)}\right) \mathbf{a} \otimes \mathbf{a}+\left(2 c_{2} I_{2}^{(1)}+c_{3} I_{1}^{(1)}+e_{2} I_{3}^{(1)}\right) \mathbf{1}+c_{4}\left(\mathbf{a} \otimes \mathbf{E}^{(1)} \cdot \mathbf{a}+\mathbf{a} \cdot \mathbf{E}^{(1)} \otimes \mathbf{a}\right)+2 c_{5} \mathbf{E}^{(1)} \\
& +e_{3}\left(\mathbf{a} \otimes \mathbf{W}^{(1)}+\mathbf{W}^{(1)} \otimes \mathbf{a}\right), \\
\mathbf{T}^{(2)}= & {\left[2 c_{1} I_{1}^{(2)}+c_{3} I_{2}^{(2)}+3 \lambda_{1}\left(I_{1}^{(1)}\right)^{2}+2 \lambda_{3} I_{1}^{(1)} I_{2}^{(1)}+\lambda_{4}\left(I_{2}^{(1)}\right)^{2}+\lambda_{5} I_{1}^{(2)}+\lambda_{7} I I_{2}^{(2)}+2 \nu_{1} I_{1}^{(1)} I_{3}^{(1)}+\nu_{2}\left(I_{3}^{(1)}\right)^{2}+\nu_{7} I I_{3}^{(2)}+\nu_{9} I I_{4}^{(2)}\right.} \\
& \left.+\nu_{14} I_{2}^{(1)} I_{3}^{(1)}\right] \mathbf{a} \otimes \mathbf{a}+\left[2 c_{2} I_{2}^{(2)}+c_{3} I_{1}^{(2)}+3 \lambda_{2}\left(I_{2}^{(1)}\right)^{2}+\lambda_{3}\left(I_{1}^{(1)}\right)^{2}+2 \lambda_{4} I_{1}^{(1)} I_{2}^{(1)}+\lambda_{6} I I_{1}^{(2)}+\lambda_{8} I I_{2}^{(2)}+2 \nu_{3} I_{2}^{(1)} I_{3}^{(1)}\right. \\
& \left.+\nu_{4}\left(I_{3}^{(1)}\right)^{2}+\nu_{8} I_{3}^{(2)}+\nu_{10} I I_{4}^{(2)}+\nu_{14} I_{1}^{(1)} I_{3}^{(1)}\right] \mathbf{1}+c_{4}\left(\mathbf{a} \otimes \mathbf{E}^{(2)} \cdot \mathbf{a}+\mathbf{a} \cdot \mathbf{E}^{(2)} \otimes \mathbf{a}\right)+\left(\lambda_{5} I_{1}^{(1)}+\lambda_{6} I_{2}^{(1)}+\nu_{5} I_{3}^{(1)}\right)\left(\mathbf{a} \otimes \mathbf{E}^{(1)} \cdot \mathbf{a}\right. \\
& \left.+\mathbf{a} \cdot \mathbf{E}^{(1)} \otimes \mathbf{a}\right)+2 c_{5} \mathbf{E}^{(2)}+2\left(\lambda_{7} I_{1}^{(1)}+\lambda_{8} I_{2}^{(2)}+\nu_{6} I_{3}^{(1)}\right) \mathbf{E}^{(1)}+\left(\nu_{9} I_{1}^{(1)}+\nu_{10} I_{2}^{(1)}+\nu_{11} I_{3}^{(1)}\right)\left(\mathbf{a} \otimes \mathbf{W}^{(1)}+\mathbf{W}^{(1)} \otimes \mathbf{a}\right) \\
& +3 \lambda_{9}\left(\mathbf{E}^{(1)}\right)^{2}+\nu_{12} \mathbf{W}^{(1)} \otimes \mathbf{W}^{(1)}+\nu_{13}\left(\mathbf{a} \otimes \mathbf{E}^{(1)} \cdot \mathbf{W}^{(1)}+\mathbf{W}^{(1)} \cdot \mathbf{E}^{(1)} \otimes \mathbf{a}+\mathbf{W}^{(1)} \otimes \mathbf{E}^{(1)} \cdot \mathbf{a}+\mathbf{a} \cdot \mathbf{E}^{(1)} \otimes \mathbf{W}^{(1)}\right), \\
\Pi^{(1)}= & -\left(2 \epsilon_{1} I_{3}^{(1)}+e_{1} I_{1}^{(1)}+e_{2} I_{2}^{(1)}\right) \mathbf{a}-2 \epsilon_{2} \mathbf{W}^{(1)}-2 e_{3} \mathbf{E}^{(1)} \cdot \mathbf{a}, \\
\Pi^{(2)}= & -\left[e_{1} I_{1}^{(2)}+e_{2} I_{2}^{(2)}+3 \mu_{1}\left(I_{3}^{(1)}\right)^{2}+\mu_{2} I_{3}^{(2)}+\nu_{1}\left(I_{1}^{(1)}\right)^{2}+2 \nu_{2} I_{3}^{(1)} I_{1}^{(1)}+\nu_{3}\left(I_{2}^{(1)}\right)^{2}+2 \nu_{4} I_{3}^{(1)} I_{2}^{(1)}+\nu_{5} I_{1}^{(2)}+\nu_{6} I I_{2}^{(2)}\right. \\
& \left.+\nu_{11} I_{4}^{(2)}+\nu_{14} I_{1}^{(1)} I_{2}^{(1)}\right] \mathbf{a}-2\left(\mu_{2} I_{3}^{(1)}+\nu_{7} I_{1}^{(1)}+\nu_{8} I_{2}^{(1)}\right) \mathbf{W}^{(1)}-2 e_{3} \mathbf{E}^{(2)} \cdot \mathbf{a}-2\left(\nu_{9} I_{1}^{(1)}+\nu_{10} I_{2}^{(1)}+\nu_{11} I_{3}^{(1)}\right) \mathbf{E}^{(1)} \cdot \mathbf{a} \\
& -2 \nu_{12} \mathbf{E}^{(1)} \cdot \mathbf{W}^{(1)}-2 \nu_{13}\left(\mathbf{E}^{(2)}\right)^{2} \cdot \mathbf{a} .
\end{aligned}
$$

Equations (15)-(19) give the second-order constitutive relations. 


\section{Small deformations and weak electric fields}

For small deformations and weak electric fields, all quadratic terms are dropped. Equations (15) reduce to

$$
\mathbf{T}=\alpha_{1} \mathbf{a} \otimes \mathbf{a}+\alpha_{2} \mathbf{1}+\mathbf{T}^{(1)}, \quad \Pi=-\beta \mathbf{a}+\Pi^{(1)},
$$

where $T^{(1)}$ and $\Pi^{(1)}$ are given by (16) and (18), respectively. We note that there are ten constants $c_{1}, c_{2}, c_{3}, c_{4}, c_{5}, \epsilon_{1}$, $\epsilon_{2}, e_{1}, e_{2}$, and $e_{3}$ for linear materials. Assuming a is oriented along the $X_{3}$ axis, from (16) and (18) we obtain

$$
\begin{aligned}
T_{11}^{(1)}= & 2\left(c_{2}+c_{5}\right) E_{11}^{(1)}+2 c_{2} E_{22}^{(1)}+\left(2 c_{2}+c_{3}\right) E_{33}^{(1)} \\
& +e_{2} W_{3}^{(1)}, \\
T_{22}^{(1)}= & 2 c_{2} E_{11}^{(1)}+2\left(c_{2}+c_{5}\right) E_{22}^{(1)}+\left(2 c_{2}+c_{3}\right) E_{33}^{(1)} \\
& +e_{2} W_{3}^{(1)}, \\
T_{33}^{(1)}= & \left(2 c_{2}+c_{3}\right) E_{11}^{(1)}+\left(2 c_{2}+c_{3}\right) E_{22}^{(1)} \\
& +2\left(c_{1}+c_{2}+c_{3}+c_{4}+c_{5}\right) E_{33}^{(1)} \\
& +\left(e_{1}+e_{2}+2 e_{3}\right) W_{3}^{(1)}, \\
T_{23}^{(1)}= & \left(c_{4}+2 c_{5}\right) E_{23}^{(1)}+e_{3} W_{2}^{(1)}, \\
T_{31}^{(1)}= & \left(c_{4}+2 c_{5}\right) E_{31}^{(1)}+e_{3} W_{1}^{(1)}, \\
T_{12}^{(1)}= & 2 c_{5} E_{12}^{(1)},
\end{aligned}
$$

and

$$
\begin{aligned}
\Pi_{1}^{(1)}= & -2 \epsilon_{2} W_{1}^{(1)}-2 e_{3} E_{31}^{(1)}, \\
\Pi_{2}^{(1)}= & -2 \epsilon_{2} W_{2}^{(1)}-2 e_{3} E_{23}^{(1)}, \\
\Pi_{3}^{(1)}= & -2\left(\epsilon_{1}+\epsilon_{2}\right) W_{3}^{(1)}-e_{2} E_{11}^{(1)}-e_{2} E_{22}^{(1)} \\
& -\left(e_{1}+e_{2}+2 e_{3}\right) E_{33}^{(1)} .
\end{aligned}
$$

Comparing (21) and (22) with the corresponding relations in the more conventional form characterized by the matrices ${ }^{12}$.

$$
\left(\begin{array}{cccccc}
c_{11} & c_{12} & c_{13} & 0 & 0 & 0 \\
c_{12} & c_{11} & c_{13} & 0 & 0 & 0 \\
c_{13} & c_{13} & c_{33} & 0 & 0 & 0 \\
0 & 0 & 0 & c_{44} & 0 & 0 \\
0 & 0 & 0 & 0 & c_{44} & 0 \\
0 & 0 & 0 & 0 & 0 & \left(c_{11}-c_{12}\right) / 2
\end{array}\right)
$$

and

$$
\left(\begin{array}{cccccc}
0 & 0 & 0 & 0 & e_{15} & 0 \\
0 & 0 & 0 & e_{15} & 0 & 0 \\
e_{31} & e_{31} & e_{33} & 0 & 0 & 0
\end{array}\right), \quad\left(\begin{array}{ccc}
\epsilon_{11} & 0 & 0 \\
0 & \epsilon_{11} & 0 \\
0 & 0 & \epsilon_{33}
\end{array}\right)
$$

we identify

$$
\begin{aligned}
& c_{1}=c_{11}-2 c_{13}+c_{33}-4 c_{44}, \quad c_{2}=c_{12} / 2, \\
& c_{3}=c_{13}-c_{12}, \quad c_{4}=-c_{11}+c_{12}+2 c_{44}, \\
& c_{5}=\left(c_{11}-c_{12}\right) / 2,
\end{aligned}
$$

$$
\begin{aligned}
& e_{1}=e_{31}+2 e_{15}-e_{33}, \quad e_{2}=-e_{31}, \quad e_{3}=-e_{15}, \\
& \epsilon_{1}=\left(\epsilon_{11}-\epsilon_{33}\right) / 2, \quad \epsilon_{2}=\left(\epsilon_{0}-\epsilon_{11}\right) / 2,
\end{aligned}
$$

where $\epsilon_{0}$, the permittivity of the vacuum appears because (22) is in terms of the polarization and the conventional form of the constitutive relation is in terms of the electric displacement.

We note that the matrices in (23) and (24) are for the crystal class $6 \mathrm{~mm}$. Transversely isotropic materials do not constitute a crystal class. It is known that within the linear constitutive theory the elastic, piezoelectric and dielectric matrices for transversely isotropic materials have the same structure as those for $6 \mathrm{~mm}$ materials. Whether the nonlinear constitutive relations for the transversely isotropic materials have the same form as those of $6 \mathrm{~mm}$ crystals remains to be studied. Nelson ${ }^{17}$ has given the specific form of the quadratic constitutive relations for $6 \mathrm{~mm}$ crystals which will be used to compare with the second-order constitutive relations for transversely isotropic materials derived above.

\section{Small deformations and strong electric fields}

Recently Tiersten ${ }^{8}$ pointed out that for piezoelectric ceramics operating under a strong driving voltage, quadratic terms like $W_{K}^{(1)} W_{L}^{(1)}$ need to be considered. In this case, we have

$$
\begin{aligned}
& \mathbf{T}=\alpha_{1} \mathbf{a} \otimes \mathbf{a}+\alpha_{2} \mathbf{1}+\mathbf{T}^{(1)}+\mathbf{T}^{(2)}, \\
& \mathbf{I}=-\beta \mathbf{a}+\mathbf{\Pi}^{(1)}+\mathbf{\Pi}^{(2)},
\end{aligned}
$$

where $T^{(1)}$ and $\Pi^{(1)}$ are the same as in (16) and (18), and

$$
\begin{aligned}
\mathbf{T}^{(2)}= & {\left[\nu_{2}\left(I_{3}^{(1)}\right)^{2}+\nu_{7} I_{3}^{(2)}\right] \mathbf{a} \otimes \mathbf{a}+\left[\nu_{4}\left(I_{3}^{(1)}\right)^{2}+\nu_{8} I I_{3}^{(2)}\right] \mathbf{1} } \\
& +\nu_{11} I_{3}^{(1)}\left(\mathbf{a} \otimes \mathbf{W}^{(1)}+\mathbf{W}^{(1)} \otimes \mathbf{a}\right)+\nu_{12} \mathbf{W}^{(1)} \otimes \mathbf{W}^{(1)},
\end{aligned}
$$

$$
\Pi^{(2)}=-\left[3 \mu_{1}\left(I_{3}^{(1)}\right)^{2}+\mu_{2} I I_{3}^{(2)}\right] \mathbf{a}-2 \mu_{2} I_{3}^{(1)} \mathbf{W}^{(1)} \text {. }
$$

We note that there are eight additional constants $\mu_{1}, \mu_{2}$, $\nu_{2}, \nu_{4}, \nu_{7}, \nu_{8}, \nu_{11}$, and $\nu_{12}$ in (27) and (28) for a nonlinear material. The constitutive relations for small deformations and strong electric fields proposed by Tiersten ${ }^{8}$ may be written as

$$
\begin{aligned}
& T_{K L}=c_{K L M N} E_{M N}^{(1)}-e_{M K L} W_{M}^{(1)}-\frac{1}{2} b_{M N K L} W_{M}^{(1)} W_{N}^{(1)}, \\
& \Pi_{K}=e_{K M N} E_{M N}^{(1)}+\chi_{K L} W_{L}^{(1)}+\frac{1}{2} \chi_{K L M} W_{L}^{(1)} W_{M}^{(1)} .
\end{aligned}
$$

In the following, we establish relations between $\mu_{1}, \mu_{2}$ and $\chi_{K L M}$, as well as relations between $\nu_{2}, \nu_{4}, \nu_{7}, \nu_{8}, \nu_{11}$, $\nu_{12}$, and $b_{M N K L}$.

\section{Relations between $\mu_{1}, \mu_{2}$, and $\chi_{A B C}$}

$\chi_{A B C}$ is fully symmetric with respect to all of its indices. Usually the last two indices are compressed into a single index like $\chi_{A \alpha}$, where the Greek index takes the values 1-6 by the following rule

$$
\begin{aligned}
& 11 \rightarrow 1, \quad 22 \rightarrow 2, \quad 33 \rightarrow 3, \\
& 23 \rightarrow 4, \quad 31 \rightarrow 5, \quad 12 \rightarrow 6 .
\end{aligned}
$$


Then the contribution of $W_{L}^{(1)} W_{M}^{(1)}$ terms to $\Pi$ in (29) can be written as

$$
\begin{aligned}
\left(\begin{array}{c}
\Pi_{1}^{(2)} \\
\Pi_{2}^{(2)} \\
\Pi_{3}^{(2)}
\end{array}\right)= & \frac{1}{2}\left(\begin{array}{llllll}
\chi_{11} & \chi_{12} & \chi_{13} & \chi_{14} & \chi_{15} & \chi_{16} \\
\chi_{21} & \chi_{22} & \chi_{23} & \chi_{24} & \chi_{25} & \chi_{26} \\
\chi_{31} & \chi_{32} & \chi_{33} & \chi_{34} & \chi_{35} & \chi_{36}
\end{array}\right) \\
& \times\left(\begin{array}{c}
\left(W_{1}^{(1)}\right)^{2} \\
\left(W_{2}^{(1)}\right)^{2} \\
\left(W_{3}^{(1)}\right)^{2} \\
2 W_{2}^{(1)} W_{3}^{(1)} \\
2 W_{3}^{(1)} W_{1}^{(1)} \\
2 W_{1}^{(1)} W_{2}^{(1)}
\end{array}\right) .
\end{aligned}
$$

For $6 \mathrm{~mm}$ crystals the matrix of $\chi$ 's has the form ${ }^{17}$

$$
\left(\begin{array}{cccccc}
0 & 0 & 0 & 0 & \chi_{15} & 0 \\
0 & 0 & 0 & \chi_{15} & 0 & 0 \\
\chi_{15} & \chi_{15} & \chi_{33} & 0 & 0 & 0
\end{array}\right) .
$$

From (28), we obtain

$$
\begin{aligned}
& \Pi_{1}^{(2)}=-2 \mu_{2} W_{3}^{(1)} W_{1}^{(1)}, \\
& \Pi_{2}^{(2)}=-2 \mu_{2} W_{3}^{(1)} W_{2}^{(1)},
\end{aligned}
$$

$\Pi_{3}^{(2)}=-\mu_{2}\left(W_{1}^{(1)}\right)^{2}-\mu_{2}\left(W_{2}^{(1)}\right)^{2}-3\left(\mu_{1}+\mu_{2}\right)\left(W_{3}^{(1)}\right)^{2}$.

Comparison of (32) and (33) gives

$\mu_{1}=\left(3 \chi_{15}-\chi_{33}\right) / 6, \quad \mu_{2}=-\chi_{15} / 2$.

\section{Relations between $\nu_{2}, \nu_{4}, \nu_{7}, \nu_{8}, \nu_{11}, \nu_{12}$ and $b_{A B C D}$}

$b_{A B C D}$ has the following symmetries

$$
b_{A B C D}=b_{B A C D}=b_{A B D C} \text {. }
$$

Usually $b_{A B C D}$ is compressed as $b_{\alpha \beta}$ which in general is not symmetric. With $b_{\alpha \beta}$, the contribution of $W_{L}^{(1)} W_{M}^{(1)}$ terms to $\mathbf{T}$ in (29) can be written as

$$
\begin{aligned}
\left(\begin{array}{c}
T_{11}^{(2)} \\
T_{22}^{(2)} \\
T_{33}^{(2)} \\
T_{23}^{(2)} \\
T_{31}^{(2)} \\
T_{12}^{(2)}
\end{array}\right)= & -\frac{1}{2}\left(\begin{array}{llllll}
b_{11} & b_{21} & b_{31} & b_{41} & b_{51} & b_{61} \\
b_{12} & b_{22} & b_{32} & b_{42} & b_{52} & b_{62} \\
b_{13} & b_{23} & b_{33} & b_{43} & b_{53} & b_{63} \\
b_{14} & b_{24} & b_{34} & b_{44} & b_{54} & b_{64} \\
b_{15} & b_{25} & b_{35} & b_{45} & b_{55} & b_{65} \\
b_{16} & b_{26} & b_{36} & b_{46} & b_{56} & b_{66}
\end{array}\right) \\
& \times\left(\begin{array}{c}
\left(W_{1}^{(1)}\right)^{2} \\
\left(W_{2}^{(1)}\right)^{2} \\
\left(W_{3}^{(1)}\right)^{2} \\
2 W_{2}^{(1)} W_{3}^{(1)} \\
2 W_{3}^{(1)} W_{1}^{(1)} \\
2 W_{1}^{(1)} W_{2}^{(1)}
\end{array}\right) .
\end{aligned}
$$

For $6 m m$ crystals the $b$ matrix is ${ }^{17}$

$$
\left(\begin{array}{cccccc}
b_{11} & b_{12} & b_{31} & 0 & 0 & 0 \\
b_{12} & b_{11} & b_{31} & 0 & 0 & 0 \\
b_{13} & b_{13} & b_{33} & 0 & 0 & 0 \\
0 & 0 & 0 & b_{44} & 0 & 0 \\
0 & 0 & 0 & 0 & b_{44} & 0 \\
0 & 0 & 0 & 0 & 0 & \left(b_{11}-b_{12}\right) / 2
\end{array}\right) .
$$

From (27), we obtain

$$
\begin{aligned}
T_{11}^{(2)}= & \left(\nu_{8}+\nu_{12}\right)\left(W_{1}^{(1)}\right)^{2}+\nu_{8}\left(W_{2}^{(1)}\right)^{2}+\left(\nu_{4}+\nu_{8}\right) \\
& \times\left(W_{3}^{(1)}\right)^{2}, \\
T_{22}^{(2)}= & \nu_{8}\left(W_{1}^{(1)}\right)^{2}+\left(\nu_{8}+\nu_{12}\right)\left(W_{2}^{(1)}\right)^{2}+\left(\nu_{4}+\nu_{8}\right) \\
& \times\left(W_{3}^{(1)}\right)^{2}, \\
T_{33}^{(2)}= & \left(\nu_{7}+\nu_{8}\right)\left(W_{1}^{(1)}\right)^{2}+\left(\nu_{7}+\nu_{8}\right)\left(W_{2}^{(1)}\right)^{2}+\left(\nu_{2}+\nu_{4}\right. \\
& \left.+\nu_{7}+\nu_{8}+2 \nu_{11}+\nu_{12}\right)\left(W_{3}^{(1)}\right)^{2}, \\
T_{23}^{(2)}= & \left(\nu_{11}+\nu_{12}\right) W_{2}^{(1)} W_{3}^{(1)}, \\
T_{31}^{(2)}= & \left(\nu_{11}+\nu_{12}\right) W_{3}^{(1)} W_{1}^{(1)}, T_{12}^{(2)}=\nu_{12} W_{1}^{(1)} W_{2}^{(1)} .
\end{aligned}
$$

The comparison of (37) with (38) yields

$$
\begin{aligned}
& \nu_{2}=\left(-b_{33}+b_{31}+b_{13}+4 b_{44}-b_{11}\right) / 2, \\
& \nu_{4}=\left(-b_{31}+b_{12}\right) / 2, \\
& \nu_{7}=\left(-b_{13}+b_{12}\right) / 2, \quad \nu_{8}=-b_{12} / 2, \\
& \nu_{11}=\left(-2 b_{44}+b_{11}-b_{12}\right) / 2, \quad \nu_{12}=\left(-b_{11}+b_{12}\right) / 2 .
\end{aligned}
$$

From (34) and (39), we conclude that the $\chi_{A B C}$ and $b_{A B C D}$ matrices for the transversely isotropic materials and $6 \mathrm{~mm}$ crystals have the same form.

\section{MATERIALS OF CLASS $\mathbf{m} m 2$}

Piezopolymers like PVDF are of class $m m 2$ symmetry.

\section{A. Form Invariant polynomlal constitutive relations}

An $m m 2$ piezoelectric material is invariant under reflections about two orthogonal planes. Let the unit normals to the two symmetry planes be $\mathbf{a}$ and $\mathbf{b}$, and $\mathbf{c}=\mathbf{a} \times \mathbf{b}$. Then any scalar polynomial function of the symmetric tensor $\mathbf{E}$ and vector $\mathbf{W}$ invariant under the above transformations must be a polynomial function of the following integrity basis ${ }^{16}$

$$
\begin{aligned}
& I_{1}=\mathbf{a} \cdot \mathbf{E} \cdot \mathbf{a}, \quad I_{2}=\mathbf{b} \cdot \mathbf{E} \cdot \mathbf{b}, \\
& I_{3}=\mathbf{c} \cdot \mathbf{E} \cdot \mathbf{c}, \quad I_{4}=\mathbf{c} \cdot \mathbf{W}, \\
& I I_{1}=J_{1}^{2}, \quad I I_{2}=J_{2}^{2}, \quad I I_{3}=J_{3}^{2}, \\
& I_{4}=(\mathbf{a} \cdot \mathbf{W})^{2}, \quad I I_{5}=(\mathbf{b} \cdot \mathbf{W})^{2}, \\
& I I_{6}=(\mathbf{a} \cdot \mathbf{W}) J_{2}, \quad I I_{7}=(\mathbf{b} \cdot \mathbf{W}) J_{1}, \\
& I I I_{1}=J_{1} J_{2} J_{3}, \quad I I I_{2}=(\mathbf{a} \cdot \mathbf{W})(\mathbf{b} \cdot \mathbf{W}) J_{3}, \\
& I I_{2}=(\mathbf{a} \cdot \mathbf{W}) J_{1} J_{3}, \quad I I_{3}=(\mathbf{b} \cdot \mathbf{W}) J_{2} J_{3},
\end{aligned}
$$


where

$J_{1}=\mathbf{b} \cdot \mathbf{E} \cdot \mathbf{c}+\mathbf{c} \cdot \mathbf{E} \cdot \mathbf{b}, \quad J_{2}=\mathbf{c} \cdot \mathbf{E} \cdot \mathbf{a}+\mathbf{a} \cdot \mathbf{E} \cdot \mathbf{c}$,

$J_{3}=\mathbf{a} \cdot \mathbf{E} \cdot \mathbf{b}+\mathbf{b} \cdot \mathbf{E} \cdot \mathbf{a}$.

With (40), a scalar polynomial function of the symmetric tensor $\mathbf{E}$ and vector $\mathbf{W}$ can be written as

$$
\begin{aligned}
\Sigma= & \Sigma\left(I_{1}, I_{2}, I_{3}, I_{4}, I I_{1}, I I_{2}, I I_{3}, I I_{4}, I I_{5}, I I_{6}, I I_{7},\right. \\
& \left.\times I I I_{1}, I I I_{2}, I I I_{3}, I I I_{4}\right) .
\end{aligned}
$$

From (5) and (42), we obtain the following general form for the polynomial constitutive relations

$$
\begin{aligned}
\mathbf{T}= & \frac{\partial \Sigma}{\partial I_{1}} \mathbf{a} \otimes \mathbf{a}+\frac{\partial \Sigma}{\partial I_{2}} \mathbf{b} \otimes \mathbf{b}+\frac{\partial \Sigma}{\partial I_{3}} \mathbf{c} \otimes \mathbf{c}+2 \frac{\partial \Sigma}{\partial I_{1}} J_{1}(\mathbf{b} \otimes \mathbf{c}+\mathbf{c} \otimes \mathbf{b})+2 \frac{\partial \Sigma}{\partial I_{2}} J_{2}(\mathbf{c} \otimes \mathbf{a}+\mathbf{a} \otimes \mathbf{c})+2 \frac{\partial \Sigma}{\partial I I_{3}} J_{3}(\mathbf{a} \otimes \mathbf{b}+\mathbf{b} \otimes \mathbf{a}) \\
& +\frac{\partial \Sigma}{\partial I_{6}}(\mathbf{a} \cdot \mathbf{W})(\mathbf{a} \otimes \mathbf{c}+\mathbf{c} \otimes \mathbf{a})+\frac{\partial \Sigma}{\partial I I_{7}}(\mathbf{b} \cdot \mathbf{W})(\mathbf{b} \otimes \mathbf{c}+\mathbf{c} \otimes \mathbf{b})+\frac{\partial \Sigma}{\partial I I_{1}}\left[J_{1} J_{2}(\mathbf{a} \otimes \mathbf{b}+\mathbf{b} \otimes \mathbf{a})+J_{2} J_{3}(\mathbf{b} \otimes \mathbf{c}+\mathbf{c} \otimes \mathbf{b})\right. \\
& \left.+J_{3} J_{1}(\mathbf{c} \otimes \mathbf{a}+\mathbf{a} \otimes \mathbf{c})\right]+\frac{\partial \Sigma}{\partial I I I_{2}}(\mathbf{a} \cdot \mathbf{W})(\mathbf{b} \cdot \mathbf{W})(\mathbf{a} \otimes \mathbf{b}+\mathbf{b} \otimes \mathbf{a})+\frac{\partial \Sigma}{\partial I I I_{3}}(\mathbf{a} \cdot \mathbf{W})\left[J_{1}(\mathbf{a} \otimes \mathbf{b}+\mathbf{b} \otimes \mathbf{a})+J_{3}(\mathbf{b} \otimes \mathbf{c}+\mathbf{c} \otimes \mathbf{b})\right] \\
& +\frac{\partial \Sigma}{\partial I I_{4}}(\mathbf{b} \cdot \mathbf{W})\left[J_{2}(\mathbf{a} \otimes \mathbf{b}+\mathbf{b} \otimes \mathbf{a})+J_{3}(\mathbf{a} \otimes \mathbf{c}+\mathbf{c} \otimes \mathbf{a})\right], \\
- & \Pi=\frac{\partial \Sigma}{\partial I_{4}} \mathbf{c}+2 \frac{\partial \Sigma}{\partial I I_{4}}(\mathbf{a} \cdot \mathbf{W}) \mathbf{a}+2 \frac{\partial \Sigma}{\partial I I_{5}}(\mathbf{b} \cdot \mathbf{W}) \mathbf{b}+\frac{\partial \Sigma}{\partial I I_{6}} J_{2} \mathbf{a}+\frac{\partial \Sigma}{\partial I I_{7}} J_{1} \mathbf{b}+\frac{\partial \Sigma}{\partial I I I_{2}} J_{3}[(\mathbf{b} \cdot \mathbf{W}) \mathbf{a}+(\mathbf{a} \cdot \mathbf{W}) \mathbf{b}]+\frac{\partial \Sigma}{\partial I I_{3}} J_{3} J_{1} \mathbf{a} \\
& +\frac{\partial \Sigma}{\partial I I I_{4}} J_{3} J_{2} \mathbf{b} .
\end{aligned}
$$

The polynomial function $\Sigma$ up to degree three is

$$
\begin{aligned}
\Sigma= & \alpha_{1} I_{1}+\alpha_{2} I_{2}+\alpha_{3} I_{3}+\beta I_{4}+c_{1} I_{1}^{2}+c_{2} I_{2}^{2}+c_{3} I_{3}^{2}+c_{4} I_{3} I_{2}+c_{5} I_{1} I_{3}+c_{6} I_{2} I_{1}+c_{7} I I_{1}+c_{8} I I_{2}+c_{9} I I_{3}+\epsilon_{1} I_{4}^{2}+\epsilon_{2} I I_{4}+\epsilon_{3} I I_{5} \\
& +e_{1} I_{1} I_{4}+e_{2} I_{2} I_{4}+e_{3} I_{3} I_{4}+e_{4} I I_{6}+e_{5} I_{7}+\lambda_{1} I_{1}^{3}+\lambda_{2} I_{2}^{3}+\lambda_{3} I_{3}^{3}+\mu_{1} I_{4}^{3}+\lambda_{4} I_{1}^{2} I_{2}+\lambda_{5} I_{1}^{2} I_{3}+\nu_{1} I_{1}^{2} I_{4}+\lambda_{6} I_{2}^{2} I_{1}+\lambda_{7} I_{2}^{2} I_{3} \\
& +\nu_{2} I_{2}^{2} I_{4}+\lambda_{8} I_{3}^{2} I_{1}+\lambda_{9} I_{3}^{2} I_{2}+\nu_{3} I_{3}^{2} I_{4}+\nu_{4} I_{4}^{2} I_{1}+\nu_{5} I_{4}^{2} I_{2}+\nu_{6} I_{4}^{2} I_{3}+\lambda_{10} I_{1} I_{1}+\lambda_{11} I_{1} I_{2}+\lambda_{12} I_{1} I_{3}+\nu_{7} I_{1} I_{4}+\nu_{8} I_{1} I I_{5} \\
& +\nu_{9} I_{1} I_{6}+\nu_{10} I_{1} I_{7}+\lambda_{13} I_{2} I_{1}+\lambda_{14} I_{2} I I_{2}+\lambda_{15} I_{2} I I_{3}+\nu_{11} I_{2} I I_{4}+\nu_{12} I_{2} I I_{5}+\nu_{13} I_{2} I_{6}+\nu_{14} I_{2} I_{7}+\lambda_{16} I_{3} I I_{1} \\
& +\lambda_{17} I_{3} I_{2}+\lambda_{18} I_{3} I_{3}+\nu_{15} I_{3} I I_{4}+\nu_{16} I_{3} I I_{5}+\nu_{17} I_{3} I_{6}+\nu_{18} I_{3} I I_{7}+\nu_{19} I_{4} I I_{1}+\nu_{20} I_{4} I_{2}+\nu_{21} I_{4} I_{3}+\mu_{2} I_{4} I I_{4} \\
& +\mu_{3} I_{4} I_{5}+\nu_{22} I_{4} I I_{6}+\nu_{23} I_{4} I_{7}+\lambda_{19} I I I_{1}+\nu_{24} I I I_{2}+\nu_{25} I I I_{3}+\nu_{26} I I_{4}+\lambda_{20} I_{1} I_{2} I_{3}+\nu_{27} I_{2} I_{3} I_{4}+\nu_{28} I_{1} I_{3} I_{4} \\
& +\nu_{29} I_{1} I_{2} I_{4}
\end{aligned}
$$

where $\alpha_{1}-\alpha_{3}$ will be shown to represent the initial stress, and $\beta$ the initial polarization. $c_{1}-c_{9}$ are elastic constants, $\epsilon_{1}-\epsilon_{3}$ dielectric constants, and $e_{1}-e_{5}$ piezoelectric constants for linear materials. $\lambda_{1}-\lambda_{20}, \mu_{1}-\mu_{3}$, and $\nu_{1}-\nu_{29}$ are constants for nonlinear materials. We note that there are 17 constants for linear materials and 52 additional constants for nonlinear materials. With (45), Eqs. (43) and (44) become

$$
\begin{aligned}
& \mathrm{T}=\left(\alpha_{1}+2 c_{1} I_{1}+c_{6} I_{2}+c_{5} I_{3}+e_{1} I_{4}+3 \lambda_{1} I_{1}^{2}+\lambda_{6} I_{2}^{2}+\lambda_{8} I_{3}^{2}+\nu_{4} I_{4}^{2}+2 \lambda_{4} I_{1} I_{2}+2 \lambda_{5} I_{1} I_{3}+2 \nu_{1} I_{1} I_{4}+\lambda_{10} I I_{1}+\lambda_{11} I I_{2}+\lambda_{12} I I_{3}\right. \\
& \left.+\nu_{7} I_{4}+\nu_{8} I I_{5}+\nu_{9} I_{6}+\nu_{10} I_{7}+\lambda_{20} I_{2} I_{3}+\nu_{28} I_{3} I_{4}+\nu_{29} I_{2} I_{4}\right) \mathbf{a} \otimes \mathbf{a}+\left(\alpha_{2}+c_{6} I_{1}+2 c_{2} I_{2}+c_{4} I_{3}+e_{2} I_{4}+\lambda_{4} I_{1}^{2}+3 \lambda_{2} I_{2}^{2}\right. \\
& +\lambda_{9} I_{3}^{2}+\nu_{5} I_{4}^{2}+2 \lambda_{6} I_{2} I_{1}+2 \lambda_{7} I_{2} I_{3}+2 \nu_{2} I_{2} I_{4}+\lambda_{13} I_{1}+\lambda_{14} I_{2}+\lambda_{15} I_{3}+\nu_{11} I_{4}+\nu_{12} I I_{5}+\nu_{13} I I_{6}+\nu_{14} I_{7}+\lambda_{20} I_{1} I_{3} \\
& \left.+\nu_{27} I_{3} I_{4}+\nu_{29} I_{1} I_{4}\right) \mathrm{b} \otimes \mathrm{b}+\left(\alpha_{3}+c_{5} I_{1}+c_{4} I_{2}+2 c_{3} I_{3}+e_{3} I_{4}+\lambda_{5} I_{1}^{2}+\lambda_{7} I_{2}^{2}+3 \lambda_{3} I_{3}^{2}+\nu_{6} I_{4}^{2}+2 \lambda_{8} I_{3} I_{1}+2 \lambda_{9} I_{3} I_{2}\right. \\
& \left.+2 \nu_{3} I_{3} I_{4}+\lambda_{16} I_{1}+\lambda_{17} I I_{2}+\lambda_{18} I_{3}+\nu_{15} I_{4}+\nu_{16} I_{5}+\nu_{17} I I_{6}+\nu_{18} I_{7}+\lambda_{20} I_{1} I_{2}+\nu_{27} I_{2} I_{4}+\nu_{28} I_{1} I_{4}\right) c \otimes c \\
& +2\left(c_{7}+\lambda_{10} I_{1}+\lambda_{13} I_{2}+\lambda_{16} I_{3}+\nu_{19} I_{4}\right) J_{1}(\mathbf{b} \otimes \mathbf{c}+\mathbf{c} \otimes \mathbf{b})+2\left(c_{8}+\lambda_{11} I_{1}+\lambda_{14} I_{2}+\lambda_{17} I_{3}+\nu_{20} I_{4}\right) J_{2}(\mathbf{c} \otimes \mathbf{a}+\mathbf{a} \otimes \mathbf{c}) \\
& +2\left(c_{9}+\lambda_{12} I_{1}+\lambda_{15} I_{2}+\lambda_{18} I_{3}+\nu_{21} I_{4}\right) J_{3}(\mathbf{a} \otimes \mathbf{b}+\mathbf{b} \otimes \mathbf{a})+\left(e_{4}+\nu_{9} I_{1}+\nu_{13} I_{2}+\nu_{17} I_{3}+\nu_{22} I_{4}\right)(\mathbf{a} \cdot \mathbf{W})(\mathbf{a} \otimes \mathbf{c}+\mathbf{c} \otimes \mathbf{a}) \\
& +\left(e_{5}+\nu_{10} I_{1}+\nu_{14} I_{2}+\nu_{18} I_{3}+\nu_{23} I_{4}\right)(\mathbf{b} \cdot \mathbf{W})(\mathbf{b} \otimes \mathbf{c}+\mathbf{c} \otimes \mathbf{b})+\lambda_{19}\left[J_{1} J_{2}(\mathbf{a} \otimes \mathbf{b}+\mathbf{b} \otimes \mathbf{a})+J_{2} J_{3}(\mathbf{b} \otimes \mathbf{c}+\mathbf{c} \otimes \mathbf{b})\right. \\
& \left.+J_{3} J_{1}(\mathbf{c} \otimes \mathbf{a}+\mathbf{a} \otimes \mathbf{c})\right]+\nu_{24}(\mathbf{a} \cdot \mathbf{W})(\mathbf{b} \cdot \mathbf{W})(\mathbf{a} \otimes \mathbf{b}+\mathbf{b} \otimes \mathbf{a})+\nu_{25}\left[J_{1}(\mathbf{a} \cdot \mathbf{W})(\mathbf{a} \otimes \mathbf{b}+\mathbf{b} \otimes \mathbf{a})+J_{3}(\mathbf{a} \cdot \mathbf{W})(\mathbf{b} \otimes \mathbf{c}+\mathbf{c} \otimes \mathbf{b})\right] \\
& +\nu_{26}\left[J_{2}(\mathbf{b} \cdot \mathbf{W})(\mathbf{a} \otimes \mathbf{b}+\mathbf{b} \otimes \mathbf{a})+J_{3}(\mathbf{b} \cdot \mathbf{W})(\mathbf{a} \otimes \mathbf{c}+\mathbf{c} \otimes \mathbf{a})\right] .
\end{aligned}
$$




$$
\begin{aligned}
-\Pi= & \left(\beta+e_{1} I_{1}+e_{2} I_{2}+e_{3} I_{3}+2 \epsilon_{1} I_{4}+\nu_{1} I_{1}^{2}+\nu_{2} I_{2}^{2}+\nu_{3} I_{3}^{2}+3 \mu_{1} I_{4}^{2}+2 \nu_{4} I_{4} I_{1}+2 \nu_{5} I_{4} I_{2}+2 \nu_{6} I_{4} I_{3}+\nu_{19} I I_{1}+\nu_{20} I I_{2}\right. \\
& \left.+\nu_{21} I I_{3}+\mu_{2} I_{4}+\mu_{3} I I_{5}+\nu_{22} I I_{6}+\nu_{23} I I_{7}+\nu_{27} I_{2} I_{3}+\nu_{28} I_{1} I_{3}+\nu_{29} I_{1} I_{2}\right) \mathbf{c}+2\left(\epsilon_{2}+\nu_{7} I_{1}+\nu_{11} I_{2}+\nu_{15} I_{3}+\mu_{2} I_{4}\right) \\
& \times(\mathbf{a} \cdot \mathbf{W}) \mathbf{a}+2\left(\epsilon_{3}+\nu_{8} I_{1}+\nu_{12} I_{2}+\nu_{16} I_{3}+\mu_{3} I_{4}\right)(\mathbf{b} \cdot \mathbf{W}) \mathbf{b}+\left(e_{4}+\nu_{9} I_{1}+\nu_{13} I_{2}+\nu_{17} I_{3}+\nu_{22} I_{4}\right) J_{2} \mathbf{a} \\
& +\left(e_{5}+\nu_{10} I_{1}+\nu_{14} I_{2}+\nu_{18} I_{3}+\nu_{23} I_{4}\right) J_{1} \mathbf{b}+\nu_{24} J_{3}[(\mathbf{b} \cdot \mathbf{W}) \mathbf{a}+(\mathbf{a} \cdot \mathbf{W}) \mathbf{b}]+\nu_{25} J_{3} J_{1} \mathbf{a}+\nu_{26} J_{3} J_{2} \mathbf{b}
\end{aligned}
$$

\section{B. Second-order theory}

We make the following expansions

$J_{1}=J_{1}^{(1)}+J_{1}^{(2)}, \quad J_{1}^{(1)}=\mathbf{b} \cdot \mathbf{E}^{(1)} \cdot \mathbf{c}+\mathbf{c} \cdot \mathbf{E}^{(1)} \cdot \mathbf{b}$,

$J_{1}^{(2)}=\mathbf{b} \cdot \mathbf{E}^{(2)} \cdot \mathbf{c}+\mathbf{c} \cdot \mathbf{E}^{(2)} \cdot \mathbf{b}$,

$J_{2}=J_{2}^{(1)}+J_{2}^{(2)}, \quad J_{2}^{(1)}=\mathbf{c} \cdot \mathbf{E}^{(1)} \cdot \mathbf{a}+\mathbf{a} \cdot \mathbf{E}^{(1)} \cdot \mathbf{c}$,

$J_{2}^{(2)}=\mathbf{c} \cdot \mathbf{E}^{(2)} \cdot \mathbf{a}+\mathbf{a} \cdot \mathbf{E}^{(2)} \cdot \mathbf{c}$,

$J_{3}=J_{3}^{(1)}+J_{3}^{(2)}, \quad J_{3}^{(1)}=\mathbf{a} \cdot \mathbf{E}^{(1)} \cdot \mathbf{b}+\mathbf{b} \cdot \mathbf{E}^{(1)} \cdot \mathbf{a}$,

$J_{3}^{(2)}=\mathbf{a} \cdot \mathbf{E}^{(2)} \cdot \mathbf{b}+\mathbf{b} \cdot \mathbf{E}^{(2)} \cdot \mathbf{a}$,

$I_{1}=I_{1}^{(1)}+I_{1}^{(2)}, \quad I_{1}^{(1)}=\mathbf{a} \cdot \mathbf{E}^{(1)} \cdot \mathbf{a}, \quad I_{1}^{(2)}=\mathbf{a} \cdot \mathbf{E}^{(2)} \cdot \mathbf{a}$,

$I_{2}=I_{2}^{(1)}+I_{2}^{(2)}, \quad I_{2}^{(1)}=\mathbf{b} \cdot \mathbf{E}^{(1)} \cdot \mathbf{b}, \quad I_{2}^{(2)}=\mathbf{b} \cdot \mathbf{E}^{(2)} \cdot \mathbf{b}$,

$I_{3}=I_{3}^{(1)}+I_{3}^{(2)}, \quad I_{3}^{(1)}=\mathbf{c} \cdot \mathbf{E}^{(1)} \cdot \mathbf{c}, \quad I_{3}^{(2)}=\mathbf{c} \cdot \mathbf{E}^{(2)} \cdot \mathbf{c}, \quad(48)$

$I_{4}=I_{4}^{(1)}, \quad I_{4}^{(1)}=\mathbf{W}^{(1)} \cdot \mathbf{c}$,

$$
\begin{array}{ll}
I I_{1}=I I_{1}^{(2)}+\ldots, & I I_{1}^{(2)}=\left(J_{1}^{(1)}\right)^{2}, \\
I I_{2}=I I_{2}^{(2)}+\ldots, & I I_{2}^{(2)}=\left(J_{2}^{(1)}\right)^{2}, \\
I I_{3}=I I_{3}^{(2)}+\ldots, & I I_{3}^{(2)}=\left(J_{3}^{(1)}\right)^{2}, \\
I I_{4}=I I_{4}^{(2)}+\ldots, & I I_{4}^{(2)}=\left(\mathbf{a} \cdot \mathbf{W}^{(1)}\right)^{2}, \\
I I_{5}=I I_{5}^{(2)}+\ldots, & I I_{5}^{(2)}=\left(\mathbf{b} \cdot \mathbf{W}^{(1)}\right)^{2}, \\
I I_{6}=I I_{6}^{(2)}+\ldots, & I I_{6}^{(2)}=\left(\mathbf{a} \cdot \mathbf{W}^{(1)}\right) J_{2}^{(1)}, \\
I I_{7}=I I_{7}^{(2)}+\ldots, & I I_{7}^{(2)}=\left(\mathbf{b} \cdot \mathbf{W}^{(1)}\right) J_{1}^{(1)} .
\end{array}
$$

Substituting (48) into (46) and (47), keeping terms up to second order, we obtain

$$
\begin{aligned}
& \mathbf{T}=\alpha_{1} \mathbf{a} \otimes \mathbf{a}+\alpha_{2} \mathbf{b} \otimes \mathbf{b}+\alpha_{3} \mathbf{c} \otimes \mathbf{c}+\mathbf{T}^{(1)}+\mathbf{T}^{(2)}, \\
& \mathbf{\Pi}=-\beta \mathbf{c}+\Pi^{(1)}+\Pi^{(2)},
\end{aligned}
$$

where

$\mathbf{T}^{(1)}=\left(2 c_{1} I_{1}^{(1)}+c_{6} I_{2}^{(1)}+c_{5} I_{3}^{(1)}+e_{1} I_{4}^{(1)}\right) \mathbf{a} \otimes \mathbf{a}+\left(c_{6} I_{1}^{(1)}+2 c_{2} I_{2}^{(1)}+c_{4} I_{3}^{(1)}+e_{2} I_{4}^{(1)}\right) \mathbf{b} \otimes \mathbf{b}+\left(c_{5} I_{1}^{(1)}+c_{4} I_{2}^{(1)}+2 c_{3} I_{3}^{(1)}+e_{3} I_{4}^{(1)}\right) \mathbf{c}$ $\otimes \mathbf{c}+2 c_{7} J_{1}^{(1)}(\mathbf{b} \otimes \mathbf{c}+\mathbf{c} \otimes \mathbf{b})+2 c_{8} J_{2}^{(1)}(\mathbf{c} \otimes \mathbf{a}+\mathbf{a} \otimes \mathbf{c})+2 c_{9} J_{3}^{(1)}(\mathbf{a} \otimes \mathbf{b}+\mathbf{b} \otimes \mathbf{a})+e_{4}(\mathbf{a} \cdot \mathbf{W})(\mathbf{a} \otimes \mathbf{c}+\mathbf{c} \otimes \mathbf{a})$

$+e_{5}(\mathbf{b} \cdot \mathbf{W})(\mathbf{b} \otimes \mathbf{c}+\mathbf{c} \otimes \mathbf{b})$,

$$
\begin{aligned}
& \mathbf{T}^{(2)}=\left[2 c_{1} I_{1}^{(2)}+c_{6} I_{2}^{(2)}+c_{5} I_{3}^{(2)}+3 \lambda_{1}\left(I_{1}^{(1)}\right)^{2}+\lambda_{6}\left(I_{2}^{(1)}\right)^{2}+\lambda_{8}\left(I_{3}^{(1)}\right)^{2}+\nu_{4}\left(I_{4}^{(1)}\right)^{2}+2 \lambda_{4} I_{1}^{(1)} I_{2}^{(1)}+2 \lambda_{5} I_{1}^{(1)} I_{3}^{(1)}+2 \nu_{1} I_{1}^{(1)} I_{4}^{(1)}\right. \\
& \left.+\lambda_{10} I I_{1}^{(2)}+\lambda_{11} I I_{2}^{(2)}+\lambda_{12} I I_{3}^{(2)}+\nu_{7} I_{4}^{(2)}+\nu_{8} I_{5}^{(2)}+\nu_{9} I I_{6}^{(2)}+\nu_{10} I I_{7}^{(2)}+\lambda_{20} I_{2}^{(1)} I_{3}^{(1)}+\nu_{28} I_{3}^{(1)} I_{4}^{(1)}+\nu_{29} I_{2}^{(1)} I_{4}^{(1)}\right] \mathbf{a} \otimes \mathbf{a} \\
& +\left[c_{6} I_{1}^{(2)}+2 c_{2} I_{2}^{(2)}+c_{4} I_{3}^{(2)}+\lambda_{4}\left(I_{1}^{(1)}\right)^{2}+3 \lambda_{2}\left(I_{2}^{(1)}\right)^{2}+\lambda_{9}\left(I_{3}^{(1)}\right)^{2}+\nu_{5}\left(I_{4}^{(1)}\right)^{2}+2 \lambda_{6} I_{2}^{(1)} I_{1}^{(1)}+2 \lambda_{7} I_{2}^{(1)} I_{3}^{(1)}+2 \nu_{2} I_{2}^{(1)} I_{4}^{(1)}\right. \\
& \left.+\lambda_{13} I I_{1}^{(2)}+\lambda_{14} I I_{2}^{(2)}+\lambda_{15} I I_{3}^{(2)}+\nu_{11} I I_{4}^{(2)}+\nu_{12} I I_{5}^{(2)}+\nu_{13} I I_{6}^{(2)}+\nu_{14} I I_{7}^{(2)}+\lambda_{20} I_{1}^{(1)} I_{3}^{(1)}+\nu_{27} I_{3}^{(1)} I_{4}^{(1)}+\nu_{29} I_{1}^{(1)} I_{4}^{(1)}\right] \mathrm{b} \\
& \otimes \mathbf{b}+\left[c_{5} I_{1}^{(2)}+c_{4} I_{2}^{(2)}+2 c_{3} I_{3}^{(2)}+\lambda_{5}\left(I_{1}^{(1)}\right)^{2}+\lambda_{7}\left(I_{2}^{(1)}\right)^{2}+3 \lambda_{3}\left(I_{3}^{(1)}\right)^{2}+\nu_{6}\left(I_{4}^{(1)}\right)^{2}+2 \lambda_{8} I_{3}^{(1)} I_{1}^{(1)}+2 \lambda_{9} I_{3}^{(1)} I_{2}^{(1)}\right. \\
& +2 \nu_{3} I_{3}^{(1)} I_{4}^{(1)}+\lambda_{16} I I_{1}^{(2)}+\lambda_{17} I I_{2}^{(2)}+\lambda_{18} I I_{3}^{(2)}+\nu_{15} I I_{4}^{(2)}+\nu_{16} I I_{5}^{(2)}+\nu_{17} I I_{6}^{(2)}+\nu_{18} I I_{7}^{(2)}+\lambda_{20} I_{1}^{(1)} I_{2}^{(1)}+\nu_{27} I_{2}^{(1)} I_{4}^{(1)} \\
& \left.+\nu_{28} I_{1}^{(1)} I_{4}^{(1)}\right] \mathbf{c} \otimes \mathbf{c}+2\left[c_{7} J_{1}^{(2)}+J_{1}^{(1)}\left(\lambda_{10} I_{1}^{(1)}+\lambda_{13} I_{2}^{(1)}+\lambda_{16} I_{3}^{(1)}+\nu_{19} I_{4}^{(1)}\right)\right](\mathbf{b} \otimes \mathbf{c}+\mathbf{c} \otimes \mathbf{b})+2\left[c_{8} J_{2}^{(2)}+J_{2}^{(1)}\left(\lambda_{11} I_{1}^{(1)}\right.\right. \\
& \left.\left.+\lambda_{14} I_{2}^{(1)}+\lambda_{17} I_{3}^{(1)}+\nu_{20} I_{4}^{(1)}\right)\right](\mathbf{c} \otimes \mathbf{a}+\mathbf{a} \otimes \mathbf{c})+2\left[c_{9} J_{3}^{(2)}+J_{3}^{(1)}\left(\lambda_{12} I_{1}^{(1)}+\lambda_{15} I_{2}^{(1)}+\lambda_{18} I_{3}^{(1)}+\nu_{21} I_{4}^{(1)}\right)\right](\mathbf{a} \otimes \mathbf{b}+\mathbf{b} \otimes \mathbf{a}) \\
& +\left(\nu_{9} I_{1}^{(1)}+\nu_{13} I_{2}^{(1)}+\nu_{17} I_{3}^{(1)}+\nu_{22} I_{4}^{(1)}\right)\left(\mathbf{a} \cdot \mathbf{W}^{(1)}\right)(\mathbf{a} \otimes \mathbf{c}+\mathbf{c} \otimes \mathbf{a})+\left(\nu_{10} I_{1}^{(1)}+\nu_{14} I_{2}^{(1)}+\nu_{18} I_{3}^{(1)}+\nu_{23} I_{4}^{(1)}\right)\left(\mathbf{b} \cdot \mathbf{W}^{(1)}\right) \\
& \times(\mathbf{b} \otimes \mathbf{c}+\mathbf{c} \otimes \mathbf{b})+\lambda_{19}\left[J_{1}^{(1)} J_{2}^{(1)}(\mathbf{a} \otimes \mathbf{b}+\mathbf{b} \otimes \mathbf{a})+J_{2}^{(1)} J_{3}^{(1)}(\mathbf{b} \otimes \mathbf{c}+\mathbf{c} \otimes \mathbf{b})+J_{3}^{(1)} J_{1}^{(1)}(\mathbf{c} \otimes \mathbf{a}+\mathbf{a} \otimes \mathbf{c})\right]+\nu_{24}\left(\mathbf{a} \cdot \mathbf{W}^{(1)}\right) \\
& \times\left(\mathbf{b} \cdot \mathbf{W}^{(1)}\right)(\mathbf{a} \otimes \mathbf{b}+\mathbf{b} \otimes \mathbf{a})+\nu_{25}\left[J_{1}^{(1)}\left(\mathbf{a} \cdot \mathbf{W}^{(1)}\right)(\mathbf{a} \otimes \mathbf{b}+\mathbf{b} \otimes \mathbf{a})+J_{3}^{(1)}\left(\mathbf{a} \cdot \mathbf{W}^{(1)}\right)(\mathbf{b} \otimes \mathbf{c}+\mathbf{c} \otimes \mathbf{b})\right] \\
& +\nu_{26}\left[J_{2}^{(1)}\left(\mathbf{b} \cdot \mathbf{W}^{(1)}\right) \times(\mathbf{a} \otimes \mathbf{b}+\mathbf{b} \otimes \mathbf{a})+J_{3}^{(1)}\left(\mathbf{b} \cdot \mathbf{W}^{(1)}\right)(\mathbf{a} \otimes \mathbf{c}+\mathbf{c} \otimes \mathbf{a})\right], \\
& \Pi^{(1)}=-\left(e_{1} I_{1}^{(1)}+e_{2} I_{2}^{(1)}+e_{3} I_{3}^{(1)}+2 \epsilon_{1} I_{4}^{(1)}\right) \mathbf{c}-2 \epsilon_{2}\left(\mathbf{a} \cdot \mathbf{W}^{(1)}\right) \mathbf{a}-2 \epsilon_{3}\left(\mathbf{b} \cdot \mathbf{W}^{(1)}\right) \mathbf{b}-e_{4} J_{2}^{(1)} \mathbf{a}-e_{5} J_{1}^{(1)} \mathbf{b},
\end{aligned}
$$




$$
\begin{aligned}
\Pi^{(2)}= & -\left[e_{1} I_{1}^{(2)}+e_{2} I_{2}^{(2)}+e_{3} I_{3}^{(2)}+\nu_{1}\left(I_{1}^{(1)}\right)^{2}+\nu_{2}\left(I_{2}^{(1)}\right)^{2}+\nu_{3}\left(I_{3}^{(1)}\right)^{2}+3 \mu_{1}\left(I_{4}^{(1)}\right)^{2}+2 \nu_{4} I_{4}^{(1)} I_{1}^{(1)}+2 \nu_{5} I_{4}^{(1)} I_{2}^{(1)}+2 \nu_{6} I_{4}^{(1)} I_{3}^{(1)}\right. \\
& \left.+\nu_{19} I I_{1}^{(2)}+\nu_{20} I I_{2}^{(2)}+\nu_{21} I I_{3}^{(2)}+\mu_{2} I I_{4}^{(2)}+\mu_{3} I I_{5}^{(2)}+\nu_{22} I I_{6}^{(2)}+\nu_{23} I I_{7}^{(2)}+\nu_{27} I_{2}^{(1)} I_{3}^{(1)}+\nu_{28} I_{1}^{(1)} I_{3}^{(1)}+\nu_{29} I_{1}^{(1)} I_{2}^{(1)}\right] \mathrm{c} \\
& -2\left(\nu_{7} I_{1}^{(1)}+\nu_{11} I_{2}^{(1)}+\nu_{15} I_{3}^{(1)}+\mu_{2} I_{4}^{(1)}\right)\left(\mathbf{a} \cdot \mathbf{W}^{(1)}\right) \mathbf{a}-2\left(\nu_{8} I_{1}^{(1)}+\nu_{12} I_{2}^{(1)}+\nu_{16} I_{3}^{(1)}+\mu_{3} I_{4}^{(1)}\right)\left(\mathbf{b} \cdot \mathbf{W}^{(1)}\right) \mathbf{b}-\left(\nu_{9} I_{1}^{(1)}\right. \\
& \left.+\nu_{13} I_{2}^{(1)}+\nu_{17} I_{3}^{(1)}+\nu_{22} I_{4}^{(1)}\right) J_{2}^{(1)} \mathbf{a}-\left(\nu_{10} I_{1}^{(1)}+\nu_{14} I_{2}^{(1)}+\nu_{18} I_{3}^{(1)}+\nu_{23} I_{4}^{(1)}\right) J_{1}^{(1)} \mathbf{b}-\nu_{24} J_{3}^{(1)}\left[\left(\mathbf{b} \cdot \mathbf{W}^{(1)}\right) \mathbf{a}+\left(\mathbf{a} \cdot \mathbf{W}^{(1)}\right) \mathbf{b}\right] \\
& -\nu_{25} J_{3}^{(1)} J_{1}^{(1)} \mathbf{a}-\nu_{26} J_{3}^{(1)} J_{2}^{(1)} \mathbf{b} .
\end{aligned}
$$

Equations (49)-(53) give the second-order constitutive relations for $m m 2$ piezoelectric materials.

\section{Small deformations and weak electric fields}

For small deformations and weak electric fields, Eqs. (49) reduce to

$$
\begin{aligned}
& \mathbf{T}=\alpha_{1} \mathbf{a} \otimes \mathbf{a}+\alpha_{2} \mathbf{b} \otimes \mathbf{b}+\alpha_{3} \mathbf{c} \otimes \mathbf{c}+\mathbf{T}^{(1)}, \\
& \mathbf{\Pi}=-\beta \mathbf{c}+\mathbf{\Pi}^{(1)},
\end{aligned}
$$

where $\mathbf{T}^{(1)}$ and $\Pi^{(1)}$ are the same as in (50) and (52). We note that there are 17 constants $c_{1}-c_{9}, \epsilon_{1}-\epsilon_{3}, e_{1}-e_{5}$ for linear materials. Assuming that unit vectors $\mathbf{a}, \mathbf{b}, \mathbf{c}$ are oriented along $X_{1}, X_{2}, X_{3}$ axes respectively, from (50) and (52) we obtain

$$
\begin{aligned}
& T_{11}^{(1)}=2 c_{1} E_{11}^{(1)}+c_{6} E_{22}^{(1)}+c_{5} E_{33}^{(1)}+e_{1} W_{3}^{(1)}, \\
& T_{22}^{(1)}=c_{6} E_{11}^{(1)}+2 c_{2} E_{22}^{(1)}+c_{4} E_{33}^{(1)}+e_{2} W_{3}^{(1)}, \\
& T_{33}^{(1)}=c_{5} E_{11}^{(1)}+c_{4} E_{22}^{(1)}+2 c_{3} E_{33}^{(1)}+e_{3} W_{3}^{(1)}, \\
& T_{23}^{(1)}=4 c_{7} E_{23}^{(1)}+e_{5} W_{2}^{(1)}, \quad T_{31}^{(1)}=4 c_{8} E_{31}^{(1)}+e_{4} W_{1}^{(1)}, \\
& T_{12}^{(1)}=4 c_{9} E_{12}^{(1)},
\end{aligned}
$$

and

$$
\begin{aligned}
& \Pi_{1}^{(1)}=-2 \epsilon_{2} W_{1}^{(1)}-2 e_{4} E_{31}^{(1)}, \\
& \Pi_{2}^{(1)}=-2 \epsilon_{3} W_{2}^{(1)}-2 e_{5} E_{23}^{(1)}, \\
& \Pi_{3}^{(1)}=-2 \epsilon_{1} W_{3}^{(1)}-e_{1} E_{11}^{(1)}-e_{2} E_{22}^{(1)}-e_{3} E_{33}^{(1)} .
\end{aligned}
$$

Comparing (55) and (56) with the corresponding relations in the more conventional form characterized by the matrices $^{10}$

$$
\left(\begin{array}{cccccc}
c_{11} & c_{12} & c_{13} & 0 & 0 & 0 \\
c_{12} & c_{22} & c_{23} & 0 & 0 & 0 \\
c_{13} & c_{23} & c_{33} & 0 & 0 & 0 \\
0 & 0 & 0 & c_{44} & 0 & 0 \\
0 & 0 & 0 & 0 & c_{55} & 0 \\
0 & 0 & 0 & 0 & 0 & c_{66}
\end{array}\right)
$$

and

$$
\left(\begin{array}{cccccc}
0 & 0 & 0 & 0 & e_{15} & 0 \\
0 & 0 & 0 & e_{24} & 0 & 0 \\
e_{31} & e_{32} & e_{33} & 0 & 0 & 0
\end{array}\right), \quad\left(\begin{array}{ccc}
\epsilon_{11} & 0 & 0 \\
0 & \epsilon_{22} & 0 \\
0 & 0 & \epsilon_{33}
\end{array}\right)
$$

we identify

$$
\begin{aligned}
& c_{1}=c_{11} / 2, \quad c_{2}=c_{22} / 2, \quad c_{3}=c_{33} / 2, \\
& c_{4}=c_{23}, \quad c_{5}=c_{31}, \quad c_{6}=c_{12}, \\
& c_{7}=c_{44} / 2, \quad c_{8}=c_{55} / 2, \quad c_{9}=c_{66} / 2, \\
& e_{1}=-e_{31}, \quad e_{2}=-e_{32}, \quad e_{3}=-e_{33}, \\
& e_{4}=-e_{15}, \quad e_{5}=-e_{24}, \\
& \epsilon_{1}=\left(\epsilon_{0}-\epsilon_{33}\right) / 2, \quad \epsilon_{2}=\left(\epsilon_{0}-\epsilon_{11}\right) / 2, \\
& \epsilon_{3}=\left(\epsilon_{0}-\epsilon_{22}\right) / 2 .
\end{aligned}
$$

\section{Small deformations and strong electric fields}

In this case, we still have equation (49) where $T^{(1)}$ and $\Pi^{(1)}$ are given by (50) and (52), respectively, and

$$
\begin{aligned}
\mathbf{T}^{(2)}= & {\left[\nu_{4}\left(I_{4}^{(1)}\right)^{2}+\nu_{7} I_{4}^{(2)}+\nu_{8} I I_{5}^{(2)}\right] \mathbf{a} \otimes \mathbf{a}+\left[\nu_{5}\left(I_{4}^{(1)}\right)^{2}\right.} \\
& \left.+\nu_{11} I I_{4}^{(2)}+\nu_{12} I I_{5}^{(2)}\right] \mathbf{b} \otimes \mathbf{b}+\left[\nu_{6}\left(I_{4}^{(1)}\right)^{2}+\nu_{15} I I_{4}^{(2)}\right. \\
& \left.+\nu_{16} I I_{5}^{(2)}\right] \mathbf{c} \otimes \mathbf{c}+\nu_{22} I_{4}^{(1)}\left(\mathbf{a} \cdot \mathbf{W}^{(1)}\right)(\mathbf{a} \otimes \mathbf{c}+\mathbf{c} \otimes \mathbf{a}) \\
& +\nu_{23} I_{4}^{(1)}\left(\mathbf{b} \cdot \mathbf{W}^{(1)}\right)(\mathbf{b} \otimes \mathbf{c}+\mathbf{c} \otimes \mathbf{b})+\nu_{24}\left(\mathbf{a} \cdot \mathbf{W}^{(1)}\right) \\
& \times\left(\mathbf{b} \cdot \mathbf{W}^{(1)}\right)(\mathbf{a} \otimes \mathbf{b}+\mathbf{b} \otimes \mathbf{a}), \\
\mathbf{I}^{(2)}= & -\left[3 \mu_{1}\left(I_{4}^{(1)}\right)^{2}+\mu_{2} I_{4}^{(2)}+\mu_{3} I I_{5}^{(2)}\right] \mathbf{c}-2 \mu_{2} I_{4}^{(1)} \\
& \times\left(\mathbf{a} \cdot \mathbf{W}^{(1)}\right) \mathbf{a}-2 \mu_{3} I_{4}^{(1)}\left(\mathbf{b} \cdot \mathbf{W}^{(1)}\right) \mathbf{b} .
\end{aligned}
$$

We note that for nonlinear materials there are fifteen constants $\mu_{1}-\mu_{3}, \nu_{4}-\nu_{8}, \nu_{11}, \nu_{12}, \nu_{15}, \nu_{16}$, and $\nu_{22}-\nu_{24}$ in (60) and (61). In the following, we establish relations between $\mu_{1}-\mu_{3}$ and $\chi_{K L M}$, and relations between $\nu_{4}-\nu_{8}, \nu_{11}$, $\nu_{12}, \nu_{15}, \nu_{16}, \nu_{22}-\nu_{24}$, and $b_{M N K L}$.

\section{Relations between $\mu_{1}-\mu_{3}$ and $\chi_{A B C}$}

For materials of class $m m 2$ we have ${ }^{17}$

$\left(\begin{array}{cccccc}0 & 0 & 0 & 0 & \chi_{15} & 0 \\ 0 & 0 & 0 & \chi_{24} & 0 & 0 \\ \chi_{15} & \chi_{24} & \chi_{33} & 0 & 0 & 0\end{array}\right)$.

From (61), we obtain

$\Pi_{1}^{(2)}=-2 \mu_{2} W_{3}^{(1)} W_{1}^{(1)}, \quad \Pi_{2}^{(2)}=-2 \mu_{3} W_{3}^{(1)} W_{2}^{(1)}$,

$\Pi_{3}^{(2)}=-\mu_{2}\left(W_{1}^{(1)}\right)^{2}-\mu_{3}\left(W_{2}^{(1)}\right)^{2}-3 \mu_{1}\left(W_{3}^{(1)}\right)^{2}$.

Comparison of (62) and (63) gives

$$
\mu_{1}=-\chi_{33} / 6, \quad \mu_{2}=-\chi_{15} / 2, \quad \mu_{3}=-\chi_{24} / 2 \text {. }
$$




\section{Relations between $\nu_{4}-\nu_{8}, \nu_{11}, \nu_{12}, \nu_{15}, \nu_{16}, \nu_{22}-\nu_{24}$, and $b_{A B C D}$}

For materials of class $m m 2$ we have ${ }^{17}$

$$
\left(\begin{array}{cccccc}
b_{11} & b_{21} & b_{31} & 0 & 0 & 0 \\
b_{12} & b_{22} & b_{32} & 0 & 0 & 0 \\
b_{13} & b_{23} & b_{33} & 0 & 0 & 0 \\
0 & 0 & 0 & b_{44} & 0 & 0 \\
0 & 0 & 0 & 0 & b_{55} & 0 \\
0 & 0 & 0 & 0 & 0 & b_{66}
\end{array}\right) .
$$

From (60), we obtain

$$
\begin{aligned}
& T_{11}^{(2)}=\nu_{7}\left(W_{1}^{(1)}\right)^{2}+\nu_{8}\left(W_{2}^{(1)}\right)^{2}+\nu_{4}\left(W_{3}^{(1)}\right)^{2}, \\
& T_{22}^{(2)}=\nu_{11}\left(W_{1}^{(1)}\right)^{2}+\nu_{12}\left(W_{2}^{(1)}\right)^{2}+\nu_{5}\left(W_{3}^{(1)}\right)^{2}, \\
& T_{33}^{(2)}=\nu_{15}\left(W_{1}^{(1)}\right)^{2}+\nu_{16}\left(W_{2}^{(1)}\right)^{2}+\nu_{6}\left(W_{3}^{(1)}\right)^{2}, \\
& T_{23}^{(2)}=\nu_{23} W_{2}^{(1)} W_{3}^{(1)}, \quad T_{31}^{(2)}=\nu_{22} W_{3}^{(1)} W_{1}^{(1)}, \\
& T_{12}^{(2)}=\nu_{24} W_{1}^{(1)} W_{2}^{(1)} .
\end{aligned}
$$

Comparison of (65) with (66) gives

$$
\begin{array}{lll}
\nu_{4}=-b_{31} / 2, & \nu_{5}=-b_{32} / 2, & \nu_{6}=-b_{33} / 2, \\
\nu_{7}=-b_{11} / 2, & \nu_{8}=-b_{21} / 2, & \nu_{11}=-b_{12} / 2, \\
\nu_{12}=-b_{22} / 2, & \nu_{15}=-b_{13} / 2, & \nu_{16}=-b_{23} / 2, \\
\nu_{22}=-b_{55} / 2, & \nu_{23}=-b_{44} / 2, & \nu_{24}=-b_{66} / 2 .
\end{array}
$$

\section{CONCLUSIONS}

By assuming that the strain energy density is a polynomial function of the symmetric strain tensor and an electric field vector, we have derived form invariant polynomial constitutive relations for nonlinear transversely isotropic and class $m m 2$ piezoelectric materials. We have also derived a second-order theory in which terms up to quadratic in the gradients of the mechanical displacement and of the electric potential are kept in the constitutive relations. Material constants appearing in the second-order theory have been identified with those in the more conventional theories.

\section{ACKNOWLEDGMENTS}

This work was supported by the U.S. Army Research Office grant DAAH 04-93-G-0214 to the University of
Missouri-Rolla, and a matching grant from the Missouri Research and Training Center. The University of MissouriRolla awarded a subcontract to the Virginia Polytechnic Institute and State University.

${ }^{1}$ K. B. Lazarus and E. F. Crawley, "Induced Strain Actuation of Composite Plates," GLT Report 197, MIT (1989).

${ }^{2}$ D. S. Norwood, M. J. Shuart, and C. T. Herakovich, "Geometrically Nonlinear Analysis of Interlaminar Stresses in Unsymmetrically Laminated Plates Subject to Inplane Mechanical Loading," in Proceedings of the ALAA/ASME/ASCE/AHS/ACS 32nd Structures, Structural Dynamics and Materials Conference (1991) (AIAA, Washington, DC), pp. 938-955.

${ }^{3}$ G. Kulkarni and S. Hanagud, "Modeling Issues in the Vibration Control with Piezoceramic Actuators," in Smart Structures and Materials, edited by G. K. Haritos and A. V. Srinivasan (ASME, New York, 1991), AD-Vol. 24, AMD-Vol. 123, pp. 7-15.

${ }^{4}$ S. Hanagud and G. Kulkarni, "Coupled Piezoceramic-Elastic Structures with Finite Deformations," in Developments in Theoretical and Applied Mechanics (Proc. of the 16th Southeastern Conference on Theoretical and Applied Mechanics), edited by B. Antar, R. Engels, A. A. Prinaris, and T. H. Moulden (The University of Tennessee Space Institute, 1992), Vol. XVI, pp. II.1.31-38.

${ }^{5}$ P. F. Pai, A. H. Nayfeh, and K. Oh, "A Nonlinear Theory of Laminated Piezoelectric Plates," in Proceedings of the 33rd ALAA/ASME/ASCE/AHS/ ASC Structures, Structural Dynamics and Materials Conference (AIAA, Washington, DC, 1992), AIAA-92-2407-CP, pp. 577-585.

${ }^{6} \mathrm{H}$. F. Tiersten, "Nonlinear Electroelastic Equations Cubic in the Small Field Variables," J. Acoust. Soc. Am. 57, 660-666 (1975).

${ }^{7}$ A. J. M. Spencer, "Theory of Invariants," in Continuum Physics I, edited by A. C. Eringen (Academic, New York, 1971), pp. 292-307.

${ }^{8}$ H. F. Tiersten, "Electroelastic Equations for Electroded Thin Plates Subject to Large Driving Voltages," J. Appl. Phys. 74 (5), 3389-3393 (1993).

${ }^{9}$ H. F. Tiersten, "Equations for the Extension and Flexure of Relatively Thin Electroelastic Plates Undergoing Large Electric Fields," in Mechanics of Electromagnetic Materials and Structures, edited by J. S. Lee, G. A. Maugin, and Y. Shindo (ASME, New York, 1993), AMD-Vol. 161, MDVol. 42, pp. 21-34.

${ }^{10}$ C. K. Lee, "Theory of Laminated Piezoelectric Plates for the Design of Distributed Sensor/Actuators. Part I: Governing Equations and Reciprocal Relations," J. Acoust. Soc. Am. 87, 1144-1158 (1992).

${ }^{11}$ T. R. Tauchert, "Piezothermalelastic Behavior of a Laminated Plate," J. Thermal Stresses 15, 25-37 (1992).

${ }^{12}$ V. V. Varadan, J.-H. Jeng, and V. K. Varadan, "Form Invariant Constitutive Relations for Transversely Isotropic Piezoelectric Materials," J. Acoust. Soc. Am. 82, 337-341 (1987).

${ }^{13} \mathrm{C}$. Truesdell and W. Noll, "The Non-Linear Field Theories of Mechanics," in Handbuch der Physik, edited by S. Flügge (Springer-Verlag, Berlin, 1965), Band III/3, pp. 229-241.

${ }^{14}$ G. A. Maugin and M. Epstein, The Electroelastic Energy-Momentum Tensor, Proc. R. Soc. London Ser. A 433, 299-312 (1991).

${ }^{15}$ Q.-S. Zheng, "On Transversely Isotropic, Orthotropic and Relatively Isotropic Functions of Symmetric Tensors, Skew-symmetric Tensors, and Vectors," Int. J. Eng. Sci. 31(10), 1399-1453 (1993).

${ }^{16}$ G. F. Smith, M. M. Smith, and R. S. Rivlin, "Integrity Bases for a Symmetric Tensor and a Vector-the Crystal Classes," Arch. Rat. Mech. Anal. 12, 93-133 (1963).

${ }^{17}$ D. F. Nelson, Electric, Optic, and Acoustic Interactions in Dielectrics (Wiley, New York, 1979), pp. 490-513. 